\title{
A Solid-State NMR Method for Solution of Zeolite Crystal Structures
}

\author{
Darren H. Brouwer, ${ }^{\dagger}$ Richard J. Darton, ${ }^{\ddagger}$ Russell E. Morris, ${ }^{\ddagger}$ and Malcolm H. Levitt ${ }^{\star}, \dagger$ \\ Contribution from the School of Chemistry, University of Southampton, Southampton, SO17 1BJ, \\ United Kingdom, and School of Chemistry, University of St. Andrews, Purdie Building,
} St. Andrews, KY16 9ST, United Kingdom

Received April 9, 2005; E-mail: mhl@soton.ac.uk

\begin{abstract}
Since zeolites are notoriously difficult to prepare as large single crystals, structure determination usually relies on powder X-ray diffraction (XRD). However, structure solution (i.e., deriving an initial structural model) directly from powder XRD data is often very difficult due to the diffraction phase problem and the high degree of overlap between the individual reflections, particularly for materials with the structural complexity of most zeolites. Here, we report a method for structure determination of zeolite crystal structures that combines powder XRD and nuclear magnetic resonance (NMR) spectroscopy in which the crucial step of structure solution is achieved using solid-state ${ }^{29} \mathrm{Si}$ double-quantum dipolar recoupling NMR, which probes the distance-dependent dipolar interactions between naturally abundant ${ }^{29} \mathrm{Si}$ nuclei in the zeolite framework. For two purely siliceous zeolite blind test samples, we demonstrate that the NMR data can be combined with the unit cell parameters and space group to solve structural models that refine successfully against the powder XRD data.
\end{abstract}

\section{Introduction}

High silica zeolites remain at the forefront of modern porous materials science. ${ }^{1-3}$ Their uses in catalysis ${ }^{4,5}$ and other emerging applications ${ }^{6}$ are intimately connected with their structural architecture. Therefore, elucidation of their crystal structures is vitally important in determining the potential of new zeolites. Since zeolites are notoriously difficult to prepare as large single crystals, structure determination usually relies on powder X-ray diffraction (XRD). However, structure solution (i.e., deriving an initial structural model) directly from powder XRD data is often very difficult, particularly for materials with the structural complexity of most zeolites.

In general, the determination of crystal structures consists of three main steps. First, unit cell parameters are determined from the reflection positions in a diffraction pattern, and possible space groups are identified from systematic absences of the reflections, perhaps with the assistance of solid-state NMR. ${ }^{7-9}$ The second and most crucial step involves deriving an initial structural model that is chemically reasonable and consistent

\footnotetext{
University of Southampton

$\doteqdot$ University of St. Andrews

(1) Corma, A.; Rey, F.; Rius, J.; Sabater, M. J.; Valencia, S. Nature 2004, 431, 287.

(2) Paillaud, J. L.; Harbuzaru, B.; Patarin, J.; Bats, N. Science 2004, 304, 990.

(3) Lee, H.; Zones, S. I.; Davis, M. E. Nature 2003, 425, 385.

(4) Corma, A.; Díaz-Cabañas, M. J.; Joaquín Martínez-Triguero, J.; Rey, F.; Rius, J. Nature 2002, 418, 514.

(5) Freyhardt, C. C.; Tsapatsis, M.; Lobo, R. F.; Balkus, K. J.; Davis, M. E. Nature 1996, 381, 295.

(6) Davis, M. E. Nature 2002, 417, 813-821.

(7) Fyfe, C. A.; Gies, H.; Kokotailo, G. T.; Marler, B.; Cox, D. E. J. Phys. Chem. 1990, 94, 3718.

(8) King, I. J.; Fayon, F.; Massiot, D.; Harris, R. K.; Evans, J. S. O. Chem. Commun. 2001, 1766.

(9) Taulelle, F. Solid State Sci. 2004, 6, 1053
}

10.1021/ja052306h CCC: $\$ 30.25$ @ 2005 American Chemical Society with available experimental data. Last, the structural model is completed by locating missing atoms and then refined, usually against diffraction data. This second step, referred to as "structure solution", is the most challenging, mainly due to the "phase problem" which arises from the fact that only intensities, and not phases, of the reflections are measured in a diffraction experiment. For materials that are limited to microcrystalline powders, such as most zeolites, structure solution is further complicated by the high degree of overlap of the reflections and other effects, such as preferred crystallite orientation, which can severely limit the reliability of intensities extracted from a powder diffraction pattern. Despite significant advances in recent years, zeolite structure solution from powder diffraction data alone ${ }^{10}$ remains challenging.

Here, we report a method for solving crystal structures of purely siliceous zeolites using a recently developed solid-state ${ }^{29} \mathrm{Si}$ double-quantum (DQ) NMR experiment, ${ }^{11}$ thereby overcoming the most difficult step of their structure determination. Figure 1 outlines the strategy for determining zeolite crystal structures using a combined XRD/NMR approach in which the unit cell parameters and space group are provided by powder XRD, while the key step of structure solution is achieved using solid-state ${ }^{29} \mathrm{Si} \mathrm{NMR}$. Once the structure is solved, it can be completed using Fourier difference maps and refined against the powder XRD pattern using the Rietveld method. The NMR structure solution method employs a robust DQ dipolar recoupling pulse sequence, ${ }^{12}$ designed using NMR symmetry prin-

(10) Burton, A. W. Z. Kristallogr. 2004, 219, 866.

(11) Brouwer, D. H.; Kristiansen, P. E.; Fyfe, C. A.; Levitt, M. H. J. Am. Chem. Soc. 2005, 127, 542.

(12) Kristiansen, P. E.; Carravetta, M.; Lai, W. C.; Levitt, M. H. Chem. Phys. Lett. 2004, 390, 1. 


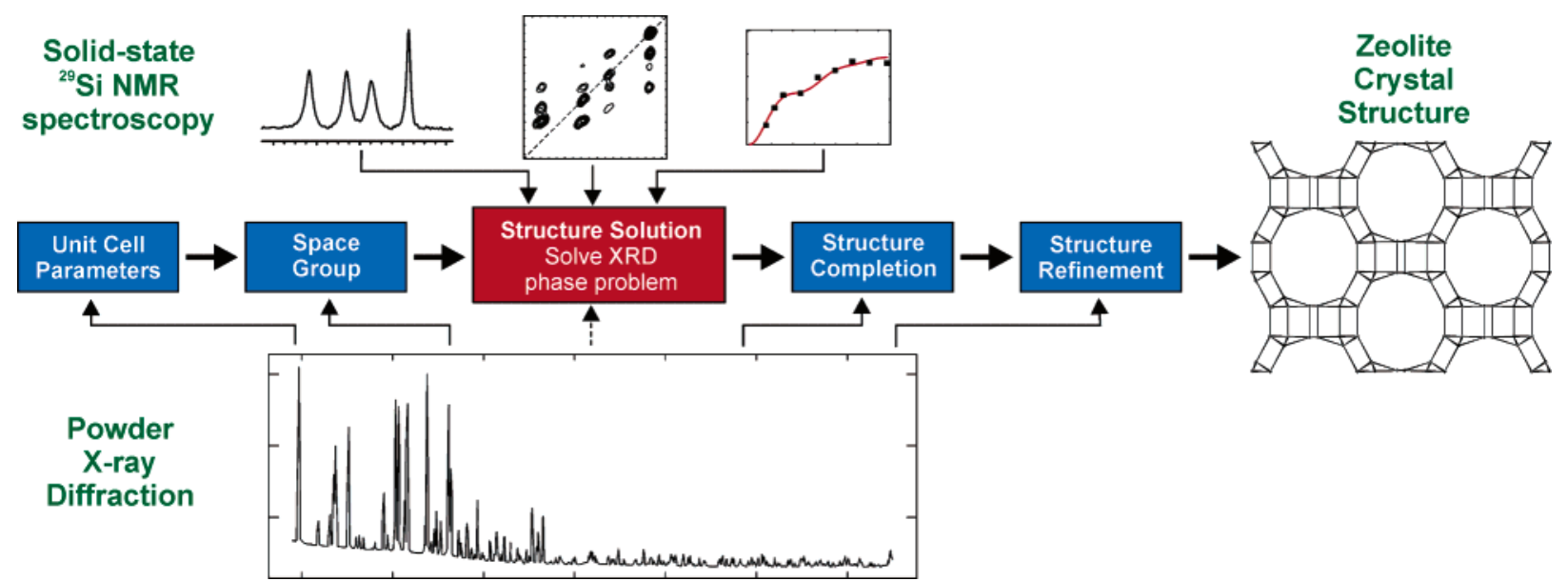

Figure 1. Overview of the structure determination strategy for zeolite crystal structures by a combination of solid-state NMR spectroscopy and powder $\mathrm{X}$-ray diffraction in which the crucial step of structure solution is accomplished using solid-state NMR.

ciples, ${ }^{13}$ with which the distance-dependent dipolar interactions between naturally abundant ${ }^{29} \mathrm{Si}$ nuclei are exploited to probe $\mathrm{Si}-\mathrm{Si}$ distances of up to $8 \AA^{11}$ under high-resolution magicangle spinning (MAS) conditions.

The strength of this XRD/NMR combination arises from the complementary nature of the techniques. Diffraction probes the long-range periodic order of the structure, whereas NMR probes the ensemble of local environments around the NMR-active nuclei. Although there are many examples of structural characterization using a combination of diffraction and NMR, $, 8,14-21$ solid-state NMR has played more of a supporting role by identifying the number, occupancies, and chemical environment of sites, assisting in the assignment of space groups, providing structural restraints, or probing dynamics and disorder, rather than being used directly for the solution of crystal structures. Solid-state NMR has been used to determine the threedimensional (3D) conformation of a folded protein in the crystalline state, where the large number of distinct sites produces numerous internuclear distance constraints. ${ }^{22}$ However, inorganic crystal structures and network materials are not amenable to this approach. Solid-state NMR has recently been used to derive a structural model of a two-dimensional (2D) layered silicate material ${ }^{20}$ and for the solution of a 3D crystal structure only in a very simple case. ${ }^{21}$

To fully test this new NMR structure solution method, a blind test was carried out in which two purely siliceous zeolite test samples, the identities of which were known only to the group at the University St. Andrews, were provided, along with unit

(13) Levitt, M. H. In Encyclopedia of Nuclear Magnetic Resonance; Grant, D. M., Harris, R. K., Eds.; John Wiley \& Sons: Chichester U.K., 2002; Vol. 9, pp 165-196.

(14) Morris, R. E.; Weigel, S. J.; Henson, N. J.; Bull, L. M.; Janicke, M. T.; Chmelka, B. F.; Cheetham, A. K. J. Am. Chem. Soc. 1994, 116, 11849

(15) van Dam, L.; Levitt, M. H. J. Mol. Biol. 2000, 304, 541.

(16) Middleton, D. A.; Peng, X.; Saunders, D.; Shankland, K.; David, W. I. F.; Markvardsen, A. J. Chem. Commun. 2002, 1976.

(17) Fyfe, C. A.; Brouwer, D. H.; Lewis, A. R.; Villaescusa, L. A.; Morris, R E. J. Am. Chem. Soc. 2002, 124, 7770.

(18) Jorda, J. L.; McCusker, L. B.; Baerlocher, C.; Morais, C. M.; Rocha, J.; Fernandez, C.; Borges, C.; Lourenco, J. P.; Ribeiro, M. F.; Gabelica, Z Microporous Mesoporous Mater. 2003, 65, 43.

(19) Harris, R. K. Solid State Sci. 2004, 6, 1025.

(20) Hedin, N.; Graf, R.; Christiansen, S. C.; Gervais, C.; Hayward, R. C.; Eckert J.; Chmelka, B. F. J. Am. Chem. Soc. 2004, 126, 9425.

(21) Dutour, J.; Guillou, N.; Huguenard, C.; Taulelle, F.; Mellot-Draznieks, C.; Ferey, G. Solid State Sci. 2004, 6, 1059.

(22) Castellani, F.; van Rossum, B.; Diehl, A.; Schubert, M.; Rehbein, K.; Oschkinat, H. Nature 2002, 420, 98. cell parameters and space groups, for NMR structural analysis to the group at the University of Southampton.

\section{Experimental Section}

Sample preparation of the zeolite blind test samples was carried out at the University of St. Andrews using procedures described in the literature. ${ }^{23-25}$ Test sample $\mathbf{1}$ was calcined to remove the organic template molecules, while test sample $\mathbf{2}$ was used in its as-synthesized form with the template molecules remaining within the zeolite channel system. The identities of these samples were known only to the group at the University of St. Andrews.

Powder X-ray diffraction analysis was carried out at the University of St. Andrews. Data $\left(7-75^{\circ} 2 \theta\right)$ were collected in transmission mode on a Stoe STADIP diffractometer equipped with a monochromator $(\mathrm{Cu}$ $\mathrm{K} \alpha, \lambda=1.54056 \AA$ ) and a position sensitive detector. The powder diffraction patterns (see Supporting Information) were indexed to give the unit cell parameters and space group of each of the blind test samples. The samples were then sent for solid-state NMR structural analysis at the University of Southampton, providing only their unit cell parameters and space groups while withholding their identities.

Solid-state NMR experiments were carried out on a Varian InfinityPlus spectrometer at a magnetic field strength of 7.0 Tesla. The ${ }^{1} \mathrm{H}$ and ${ }^{29} \mathrm{Si}$ frequencies were -299.85 and $59.56 \mathrm{MHz}$, respectively. A $6 \mathrm{~mm}$ MAS probe was used with the rotor holding approximately $150 \mathrm{mg}$ of sample. All experiments were carried out a spinning frequency of $4000 \pm 2 \mathrm{~Hz}$.

The pulse sequence diagram of the two-dimensional ${ }^{29} \mathrm{Si}$ doublequantum NMR experiment ${ }^{11}$ used to probe distances between ${ }^{29} \mathrm{Si}-{ }^{29} \mathrm{Si}$ spin pairs is displayed in Figure 2. The experiment employs the robust symmetry-based ${ }^{13} \mathrm{SR} 26_{4}{ }^{11}$ homonuclear dipolar recoupling sequence $^{12}$ to excite and reconvert DQ coherences between dipolar coupled pairs of ${ }^{29} \mathrm{Si}$ nuclei. The implementation of this pulse sequence is described in detail in the Supporting Information.

Solid-state NMR experiments were carried out on test sample 1 without ${ }^{1} \mathrm{H} \rightarrow{ }^{29} \mathrm{Si} \mathrm{CP}$ over a period of 5 days, while the experiments for test sample 2 were performed over a period of 3 days and employed ${ }^{1} \mathrm{H} \rightarrow{ }^{29} \mathrm{Si} \mathrm{CP}$ to take advantage of the presence of occluded organic template molecules in the zeolite channel system. The full details of the NMR experiments are provided in the Supporting Information.

The relative occupancies of the $\mathrm{Si}$ sites were established by evaluating the relative peak areas obtained in deconvolutions of the

(23) Barrett, P. A.; Camblor, M. A.; Corma, A.; Jones, R. H.; Villaescusa, L. A. Chem. Mater. 1997, 9, 1713

(24) Kuperman, A.; Oliver, S.; Ozin, G. A.; Garces, J. M.; Olken, M. M. Nature 1993, 365, 239

(25) Bull, I.; Lightfoot, P.; Villaescusa, L. A.; Bull, L. M.; Gover, R. K. B.; Evans, J. S. O.; Morris, R. E. J. Am. Chem. Soc. 2003, 125, 4342 


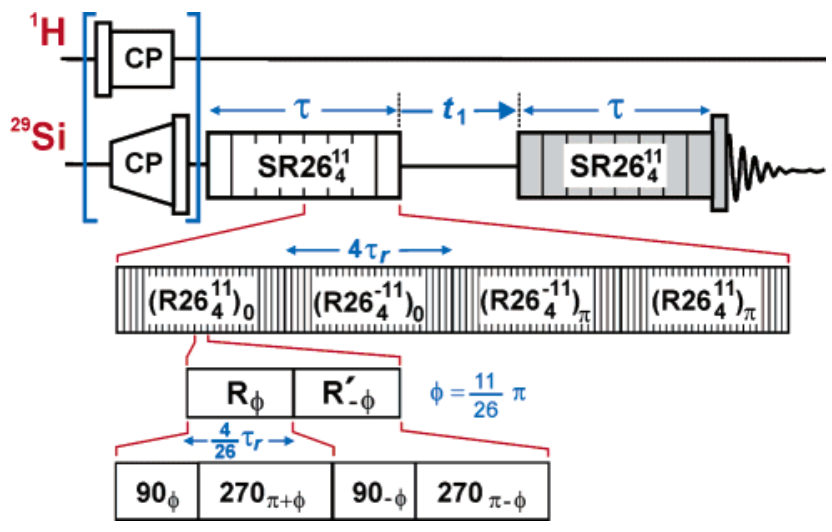

Figure 2. Pulse sequence diagram for two-dimensional ${ }^{29}$ Si double-quantum correlation spectroscopy using the symmetry-based SR $26_{4}{ }^{11}$ homonuclear dipolar recoupling sequence. ${ }^{11,12}$ The DQ recoupling time is denoted by $\tau$.

1D ${ }^{29} \mathrm{Si}$ spectra. The intersite connectivities were established by evaluating the relative intensities of the correlations in the 2D DQ correlation spectrum obtained with a recoupling time of $6 \mathrm{~ms}$. The DQ curves were obtained by extracting the amplitudes of the correlation peaks in a series of $2 \mathrm{D}$ spectra (with $\mathrm{DQ}$ recoupling times from 4 to $32 \mathrm{~ms}$ ) and plotting these amplitudes as functions of the DQ recoupling time, after scaling with respect to the peak amplitudes obtained in a corresponding 1D spectrum.

Structure solution of the crystal structures of the zeolite blind test samples from solid-state ${ }^{29} \mathrm{Si}$ NMR data was accomplished using the algorithm outlined below and described in detail in the Supporting Information. This structure solution algorithm, as well as the deconvolution of 1D and 2D NMR spectra, was implemented as Mathematica (version 5.1 $)^{26}$ notebooks and run on a Linux machine equipped with an AMD $2.0 \mathrm{GHz}$ processor and $1 \mathrm{~GB}$ of RAM.

Structure completion and refinements of the zeolite crystal structures against the powder XRD data were carried out using the atomic coordinates for the silicon atoms solved by solid-state NMR as the initial structural models. For test sample 1, the missing oxygen atoms were located from Fourier difference maps using phases calculated from the NMR structure. For test sample 2, the oxygen atoms were placed geometrically between $\mathrm{Si}$ atoms, and the zeolite framework was optimized using the distance least-squares method ${ }^{27}$ prior to Rietveld refinement, while the carbon atoms of the template molecules were located from Fourier difference maps. The full structure completion and refinement details are provided in the Supporting Information. Structure solution directly from powder XRD using the EXPO program ${ }^{28}$ was also attempted for both test samples, and these details are also provided in Supporting Information.

\section{Results and Discussion}

The first blind test zeolite sample, referred to as test sample 1, was measured by powder XRD to have the unit cell parameters $a=18.669, b=13.503, c=7.662 \AA$. $\beta=102.1^{\circ}$, and the monoclinic space group $12 / \mathrm{m}$. The ${ }^{29} \mathrm{Si}$ MAS NMR spectrum of test sample 1 (Figure 3a) consists of four resolved peaks with equal intensities, revealing that there are four $\mathrm{Si}$ sites in the crystal structure with equal site occupancies. The chemical shifts indicate that the sites are in tetrahedral $\mathrm{Si}(\mathrm{OSi})_{4}$ environments. A $2 \mathrm{D}{ }^{29} \mathrm{Si} \mathrm{DQ}$ correlation spectrum, obtained with a DQ

(26) Wolfram, S. Mathematica: A System for Doing Mathematics by Computer, version 5.1; Wolfram Media: Champaign, IL, 2004.

(27) Baerlocher, Ch.; Hepp, A.; Meier, W. M. DLS-76: A Program for the Simulation of Crystal Structures by Geometric Refinement; Lab. f. Kristallographie, ETH: Zurich, 1978.

(28) Altomare, A.; Burla, M. C.; Camalli, M.; Carrozzini, B.; Cascarano, G L.; Giacovazzo, C.; Guagliardi, A.; Moliterni, A. G. G.; Polidori, G.; Rizzi, R. J. Appl. Crystallogr. 1999, 32, 339.
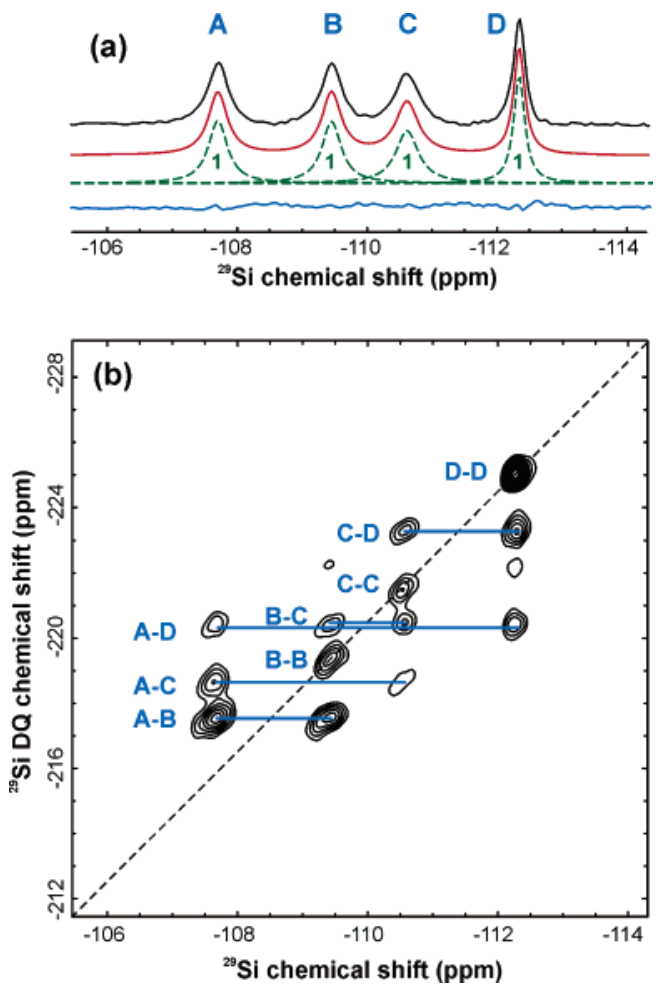

Figure 3. One- and two-dimensional solid-state ${ }^{29} \mathrm{Si}$ NMR spectra for zeolite test sample 1. (a) Deconvolution of a quantitative 1D ${ }^{29} \mathrm{Si}$ MAS NMR spectrum (from top to bottom: experimental spectrum, calculated spectrum, individual peaks with relative peak areas indicated, difference spectrum). (b) Two-dimensional ${ }^{29} \mathrm{Si} \mathrm{DQ}$ correlation spectrum obtained with a recoupling time of $6 \mathrm{~ms}$.

recoupling time of $6 \mathrm{~ms}$, is presented in Figure 3b. Correlations between pairs of peaks appear in the indirect dimension at the sum of their isotropic chemical shifts. With a sufficiently short recoupling time, the DQ correlation signals arise primarily from dipolar coupled ${ }^{29} \mathrm{Si}-{ }^{29} \mathrm{Si}$ spin pairs across $\mathrm{Si}-\mathrm{O}-\mathrm{Si}$ linkages and, therefore, reveal the $\mathrm{Si}-\mathrm{O}-\mathrm{Si}$ intersite connectivities.

With the exception of the auto-correlations, ${ }^{11,20}$ connectivity information of this kind has been available for some time from NMR experiments exploiting ${ }^{29} \mathrm{Si}-\mathrm{O}-{ }^{29} \mathrm{Si} J$-couplings. ${ }^{29,30}$ However, an invaluable feature of this dipolar recoupling NMR experiment is that additional structural information in the form of longer range $\mathrm{Si}-\mathrm{Si}$ distances can be probed by collecting a series of 2D DQ correlation spectra with different recoupling times. The resulting "double-quantum curves" (intensities of the DQ correlation peaks as functions of the recoupling time) are highly sensitive to $\mathrm{Si}-\mathrm{Si}$ distance distributions and can be faithfully simulated given a set of $\mathrm{Si}-\mathrm{Si}$ distances of up to 8 $\AA .{ }^{11}$ The set of experimental DQ curves for test sample $\mathbf{1}$ is presented in Figure 4.

The solution of the zeolite crystal structure from this solidstate ${ }^{29} \mathrm{Si}$ NMR data was carried out using an algorithm developed to find the set of $\mathrm{Si}$ atomic coordinates in the asymmetric unit, which minimize the sum of squares of the residuals between the experimental DQ curves and the DQ curves calculated from the set of $\mathrm{Si}-\mathrm{Si}$ distances for a given arrangement of Si atoms. The algorithm, described schematically in Figure 5, combines a "grid search" with subsequent least-

(29) Fyfe, C. A.; Gies, H.; Feng, Y.; Kokotailo, G. T. Nature 1989, 341, 223.

(30) Fyfe, C. A.; Feng, Y.; Grondey, H.; Kokotailo, G. T.; Gies, H. Chem. Rev. 1991, 91,1525 


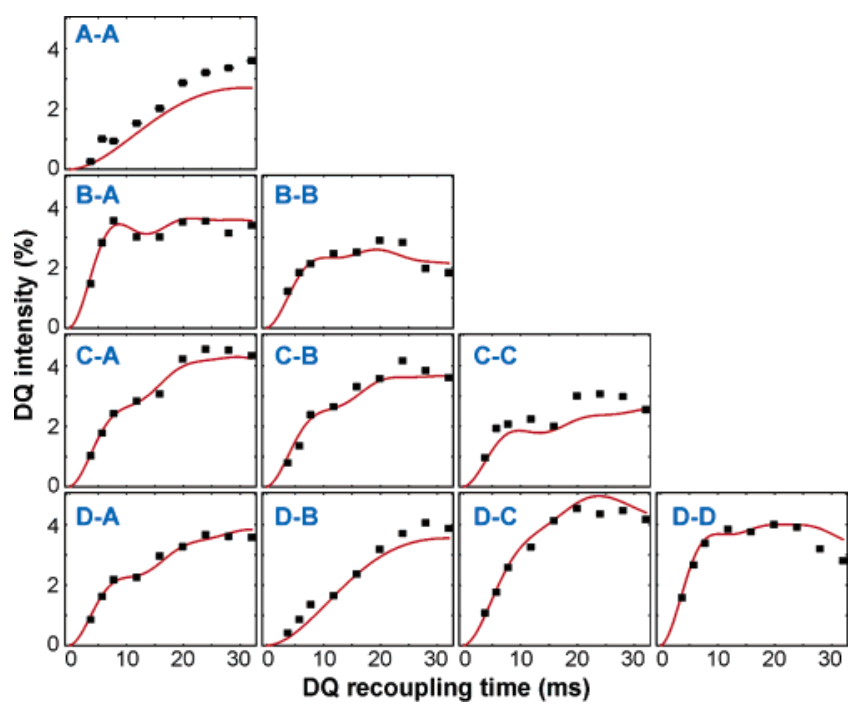

Figure 4. ${ }^{29} \mathrm{Si} \mathrm{DQ}$ curves for test sample $\mathbf{1}$ in which the experimental data (black squares) were obtained by integrating the correlation peaks in a series of 2D ${ }^{29} \mathrm{Si}$ DQ correlation spectra and were scaled with respect to the signal intensities in the $1 \mathrm{D}$ spectrum. The DQ curves calculated from the structure solved by NMR are displayed as red lines.

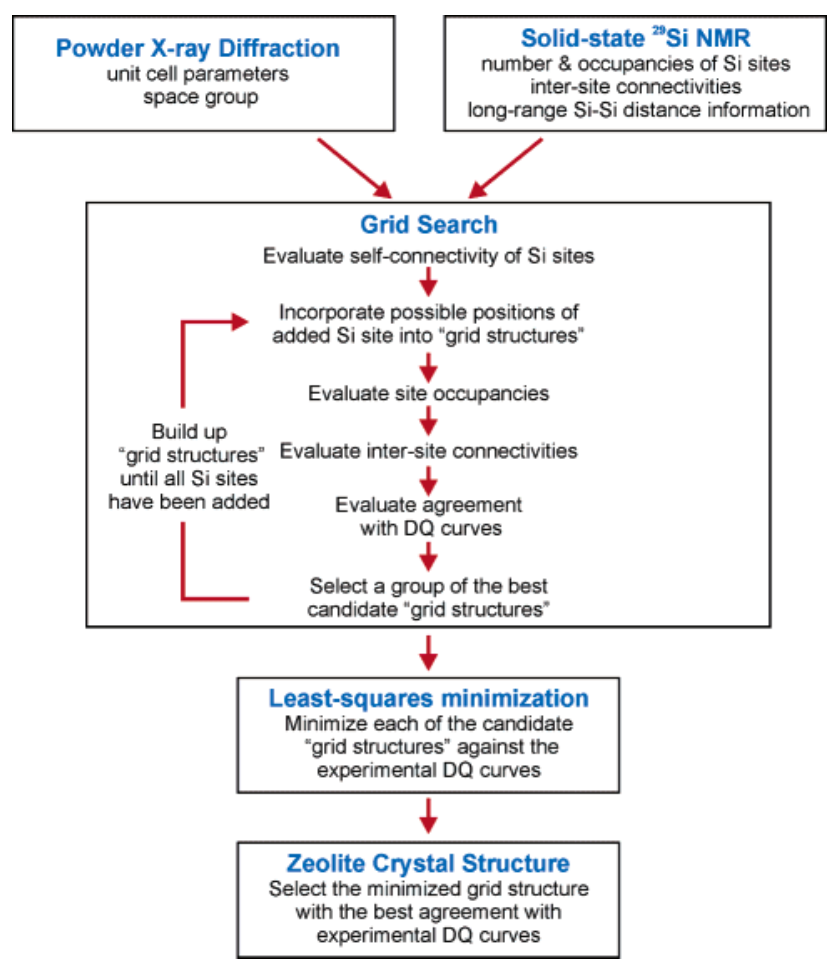

Figure 5. Schematic description of the algorithm for solution of zeolite crystal structures from solid-state ${ }^{29} \mathrm{Si}$ NMR data. The full details of the algorithm are presented in the Supporting Information.

squares minimization. The grid search consisted of building up candidate structures one $\mathrm{Si}$ site at a time with the atomic coordinates restricted to points on a three-dimensional grid of the asymmetric unit. The possible positions on the grid for the $\mathrm{Si}$ site to be added to the candidate structures were first evaluated and limited to those for which the connectivity between symmetry-related positions of the same Si site was satisfied. For example, the intensity of the auto-correlation in the 2D ${ }^{29} \mathrm{Si}$ DQ correlation spectrum of test sample 1 (Figure $3 \mathrm{~b}$ ) indicates that site $\mathrm{D}$ is connected to two symmetry-related positions of site D. The grid positions which satisfy the "self-
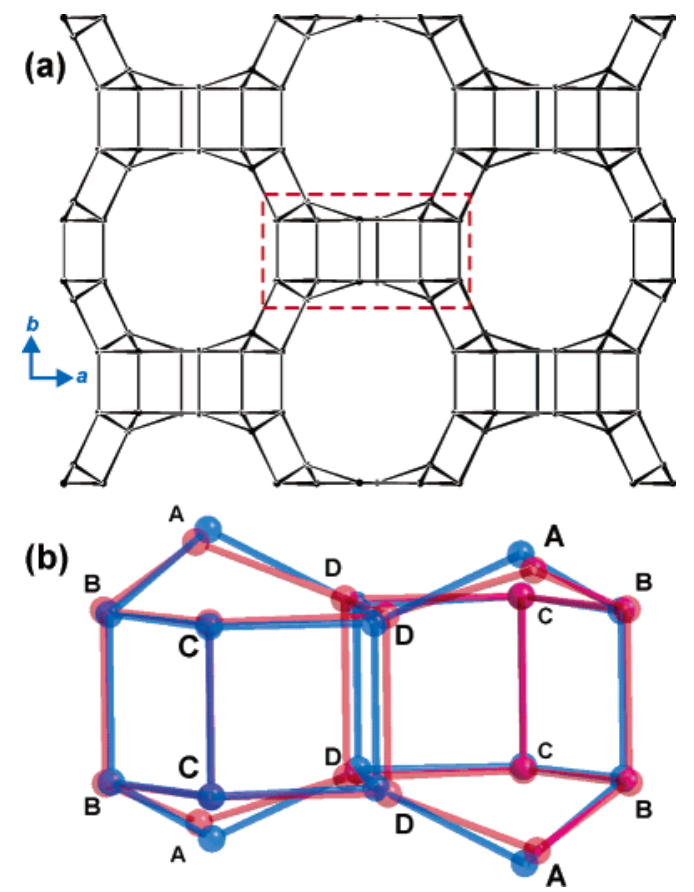

Figure 6. Structure of zeolite test sample 1 solved by solid-state NMR. (a) Projection along the straight channels ( $c$-axis) with the fused-cage pair highlighted. (b) Comparison of the structure solved by solid-state NMR (red) to the structure obtained after completion and refinement against powder XRD data (blue). Only the silicon atoms are shown.

connectivity" for the added Si site were then incorporated into each of the candidate structures. These new candidate structures were evaluated to ensure that the relative occupancies of the sites were consistent with the relative intensities of the peaks in the $1 \mathrm{D}{ }^{29} \mathrm{Si}$ MAS NMR spectrum, and that the connectivities between the sites were in agreement with the intersite connectivities derived from the $2 \mathrm{D}{ }^{29} \mathrm{Si} \mathrm{DQ}$ correlation spectrum. For each of the new candidate structures which meet the occupancy and connectivity criteria, the quality of fits to the relevant experimental DQ curves was then evaluated, and a group of structures with the best agreement was selected. The next $\mathrm{Si}$ site was added to this group of candidate structures as described above, and the whole process was repeated until all of the $\mathrm{Si}$ sites had been incorporated into the candidate structures. Each of these candidate "grid structures" was then subjected to leastsquares minimization against the set of experimental DQ curves, with the $\mathrm{Si}-\mathrm{Si}$ distances between $\mathrm{Si}$ atoms known to share a $\mathrm{Si}-\mathrm{O}-\mathrm{Si}$ linkage restrained (softly) to be $3.1 \AA$, as the distances across $\mathrm{Si}-\mathrm{O}-\mathrm{Si}$ linkages are known to fall within the narrow range of about 3.03.2 $\AA$. This combination of a grid search and least-squares minimization should ensure that the global minimum structure is found. This algorithm is described in full detail in the Supporting Information.

The structure for test sample 1 found by the algorithm to give the best agreement with the NMR data is presented in Figure 6. The DQ curves calculated from this structure are displayed in Figure 4 and are in excellent agreement with the experimental data.

Two important aspects contribute to the viability of this NMR structure solution method. First, there exists an analytical expression for the DQ curves for isolated pairs of dipolar coupled nuclei ${ }^{31,32}$ (see Supporting Information). Second, the

(31) Mueller, K. T. J. Magn. Reson. A 1995, 113, 81. 

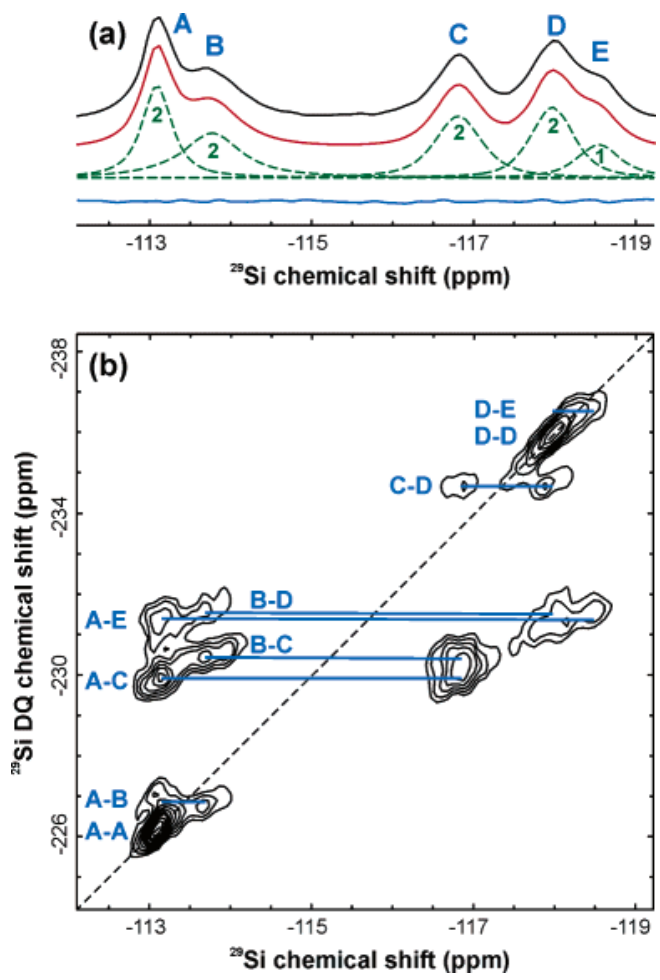

Figure 7. One- and two-dimensional solid-state ${ }^{29} \mathrm{Si}$ NMR spectra for zeolite test sample 2. (a) Deconvolution of a quantitative 1D ${ }^{29} \mathrm{Si}$ MAS NMR spectrum (from top to bottom: experimental spectrum, calculated spectrum, individual peaks with relative peak areas indicated, difference spectrum). (b) Two-dimensional ${ }^{29} \mathrm{Si}$ DQ correlation spectrum obtained with a recoupling time of $6 \mathrm{~ms}$.

natural abundance of ${ }^{29} \mathrm{Si}(4.7 \%)$ is high enough that there are significant numbers of isolated ${ }^{29} \mathrm{Si}-{ }^{29} \mathrm{Si}$ spin pairs, yet low enough that complications arising from multispin clusters are negligible. Consequently, the full DQ curves can be constructed by summing sets of DQ curves calculated for isolated ${ }^{29} \mathrm{Si}-{ }^{29} \mathrm{Si}$ spin pairs. For these reasons, the evaluation of candidate structures by comparing experimental and calculated DQ curves can be carried out very rapidly.

The solid-state NMR structure for test sample $\mathbf{1}$ was used as the starting model for refinement against the powder XRD data, with the remaining oxygen atoms located from Fourier maps. As Figure 6b demonstrates, the structure solved by NMR is essentially identical to the structure obtained after completion and successful refinement against the XRD data. A comparison with the known zeolite topologies ${ }^{33}$ revealed that this sample was ITQ-4 ${ }^{23}$ (IFR topology). The solution of this structure by NMR was reasonably straightforward due to its relatively low complexity. Indeed, the structure of ITQ-4 was originally solved using powder XRD, ${ }^{23}$ and the structure of test sample $\mathbf{1}$ could be solved using the powder diffraction program $\mathrm{EXPO}^{28}$ without using NMR.

The second zeolite test sample provided a more challenging case. Powder XRD of test sample 2 provided the unit cell parameters $a=7.407, b=14.017, c=18.670 \AA$, and the orthorhombic space group Pnnm. The sample was in its assynthesized form with the organic template molecules occluded

(32) Mueller, K. T.; Jarvie, T. P.; Aurentz, D. J.; Roberts, B. W. Chem. Phys. Lett. 1996, 242, 535.

(33) International Zeolite Association. Atlas of Zeolite Framework Types, Baerlocher, Ch., Meier, W. M., Olson, D. H., Eds.; Elsevier: Amsterdam, The Netherlands, 2001.

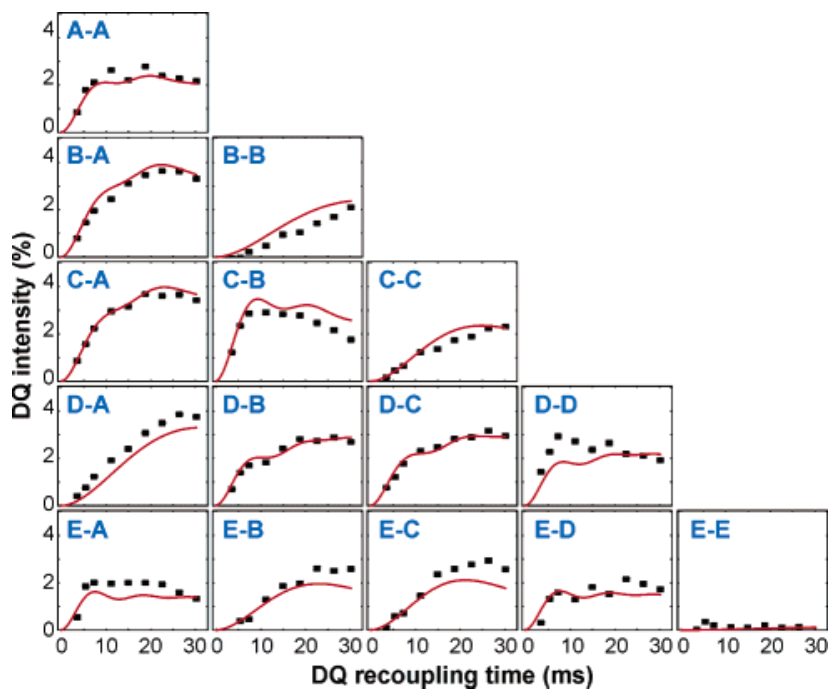

Figure 8. ${ }^{29} \mathrm{Si}$ DQ curves for test sample $\mathbf{2}$ in which the experimental data (black squares) were obtained by integrating the correlation peaks in a series of $2 \mathrm{D}^{29} \mathrm{Si} \mathrm{DQ}$ correlation spectra and were scaled with respect to the signal intensities in the 1D spectrum. The DQ curves calculated from the structure solved by NMR are displayed as red lines.

in the zeolite channels. We were not able to solve the structure using standard powder XRD techniques due to unreliable XRD intensities arising from the added complexity of occluded organic templates and severe preferred orientation effects resulting from the platelike crystal morphology.

The ${ }^{1} \mathrm{H} \rightarrow{ }^{29} \mathrm{Si}$ cross-polarization (CP) MAS NMR spectrum of test sample 2 (Figure 7a) indicates there are five tetrahedral $\mathrm{Si}$ sites in the crystal structure with relative occupancies of $2: 2: 2: 2: 1$. The chemical shifts confirm that the sites are in tetrahedral $\mathrm{Si}(\mathrm{OSi})_{4}$ environments. The $2 \mathrm{D}{ }^{29} \mathrm{Si} \mathrm{DQ}$ correlation spectrum in Figure $7 \mathrm{~b}$ reveals the intersite connectivities. The set of experimental DQ curves, derived from a series of 2D DQ correlation spectra, is presented in Figure 8.

The structure for test sample 2 found by the NMR structure solution algorithm to give the best agreement with the ${ }^{29} \mathrm{Si}$ solidstate NMR data is presented in Figure 9. To provide a complete structure for refinement against the powder XRD data, oxygen atoms were added midway between $\mathrm{Si}$ atoms, and the geometry of the zeolite framework was optimized with the distance leastsquares method, ${ }^{27}$ while the atoms of the template molecules were located using Fourier maps. As Figure 9b demonstrates, the structure solved by NMR is, again, essentially the same as the structure obtained after completion and successful refinement against the powder XRD data. A comparison with the known zeolite topologies ${ }^{33}$ revealed that this sample was ferrierite ${ }^{14}$ (FER topology). This is the first time that solid-state NMR has been used to solve the $3 \mathrm{D}$ crystal structure of a sample that was intractable by powder XRD alone.

\section{Conclusion}

The solid-state NMR method described here correctly solved the crystal structures of both zeolite blind test samples, illustrating the potential of this combined NMR/XRD approach for even the more difficult cases. A key requirement for this method in its present form is that the different crystallographic sites must give rise to distinct NMR signals and, consequently, spectral resolution can be a limiting factor. For example, the structure solution of most aluminosilicate zeolites would not 


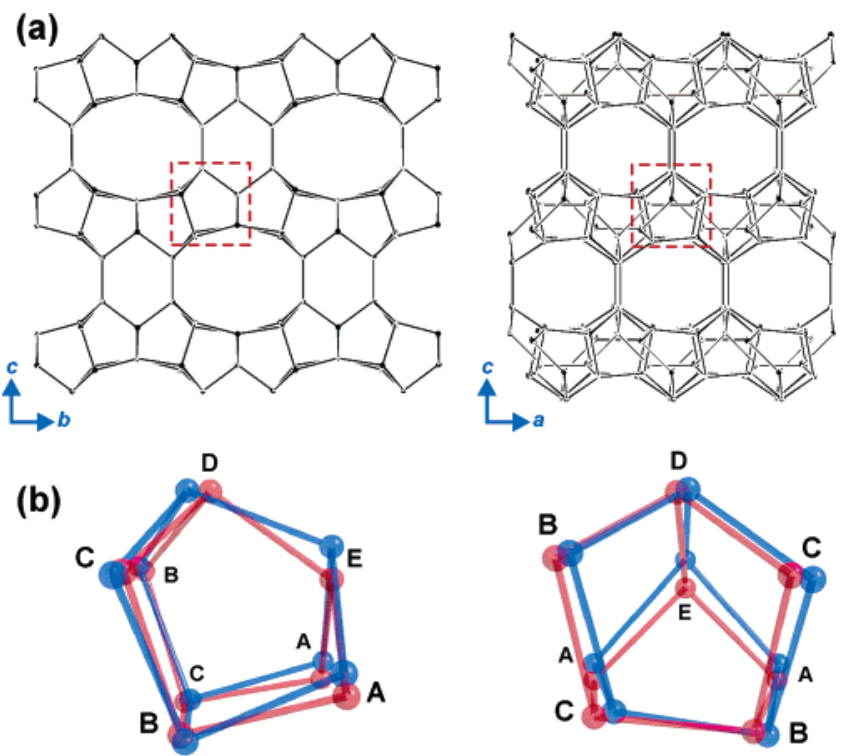

Figure 9. Structure of zeolite test sample 2 solved by solid-state NMR. (a) Projection along the $a$-axis (left) and $b$-axis (right) of the structure solved by solid-state NMR. (b) Comparison of the structure solved by solid-state NMR (red) to the structure obtained after completion and refinement against powder XRD data (blue).

be amenable to this NMR approach since the crystallographic sites are usually not distinguishable by NMR. However, with advances in zeolite synthesis methods, ${ }^{34}$ many new zeolites of significant importance can be synthesized as purely siliceous materials that give highly resolved ${ }^{29} \mathrm{Si}$ NMR spectra, and this work demonstrates that solid-state NMR can play a vital role in their structural characterization. Further improvements are under development to deal with cases in which a number of silicon sites may give rise to overlapping peaks in the ${ }^{29} \mathrm{Si}$ spectrum (for example, ref 29). A second potential limitation of this method in its current form is that the space group is determined from the powder XRD pattern and can sometimes be in conflict with the solid-state NMR data. Therefore, the information provided by both XRD and NMR should always be used in combination when ascertaining possible space groups. An approach to extend this NMR structure solution technique so that the space group is not necessarily required as an input is under development.

Although purely siliceous zeolites represent a relatively favorable case for NMR characterization, it is anticipated that the method should also be applicable, perhaps in a modified form, to more complex inorganic materials and also isotopically enriched organic molecules. Furthermore, it is anticipated that the range and accuracy of the method will be greatly extended by incorporating chemical shift ${ }^{35}$ and energy ${ }^{36}$ calculations in the structure solution and refinement stages. While this technique is unlikely to replace XRD for most applications, where XRD has specific problems (such as extreme preferred orientation or disordered layered structures ${ }^{20}$ ), structure solution by solid-state NMR may prove to be a very valuable technique. In summary, we claim that this work establishes a new paradigm in the combination of diffraction and spectroscopic methods and, in addition to structure solution, offers up the possibility of a new diffraction/NMR refinement technique in which structures are refined against both diffraction and NMR simultaneously.

Acknowledgment. We acknowledge Per Eugen Kristiansen for developing the dipolar recoupling NMR pulse sequence, and Ole Johannessen for assistance with the NMR experiments. D.H.B. thanks the Natural Sciences and Engineering Research Council of Canada for a Postdoctoral Fellowship. R.E.M. thanks the Royal Society for the provision of a University Research Fellowship, and M.H.L. thanks the EPSRC (UK) for financial support.

Supporting Information Available: Details of the calculation of DQ curves and the NMR structure solution algorithm, additional details of solid-state NMR and powder XRD experiments, and full details of the structure solution, completion, and refinement of the two zeolite blind test samples. This material is available free of charge via the Internet at http://pubs.acs.org.

\section{JA052306H}

(34) Camblor, M. A.; Villaescusa, L. A.; Díaz-Cabañas, M. J. Top. Catal. 1999 9, 59.

(35) Pickard, C. J.; Mauri, F. Phys. Rev. B 2001, 63, 245101.

(36) Gale, J. D.; Rohl, A. L. Mol. Simul. 2003, 29, 291. 